Punjab University Journal of Mathematics (2021),53(10),711-728

https://doi.org/10.52280/pujm.2021.531002

\title{
Soliton solutions of coupled complex modified Korteweg-de Vries system through Binary Darboux transformation
}

\author{
Zaheer Abbas \\ Department of Physics, \\ The University of Lahore, Lahore, Pakistan, \\ Email: Chzaheer960@gmail.com \\ Nosheen Mushahid \\ Department of Physics, \\ The University of Lahore, Lahore, Pakistan, \\ Email: nosheen.mushahid@gmail.com
}

Received: 06 May, 2020 / Accepted: 27 August, 2021 / Published online: 26 December, 2021

\begin{abstract}
In this article, we find various kind of solutions of coupled complex modified $(\mathrm{KdV})$ system by using very interesting method binary Darboux transformation. Generally the solutions are classified into zero seed and non-zero seed. In zero seed solutions, we find breather solution and one soliton solution. While in non-zero seed solutions, we obtain bright-bright solitons, w-shaped solitons, bright-dark solitons, periodic and rouge waves solutions. The behavior of these solutions can easily examine from figures.
\end{abstract}

AMS (MOS) Subject Classification Codes: 35S29; 40S70; $25 \mathrm{U} 09$

Key Words: Binary Darboux transformation, Breather Solutions, Soliton Solutions, Periodic Solutions, Rouge Waves, Coupled complex modified Korteweg-de Vries

\section{INTRODUCTION}

In nonlinear science, integrable models play an essential role to describe the wave propagation in nonlinear media. These integrable systems have a number of applications in different phenomenons like water wave, signal transmission in optical fibers, magnetics, molecular biology and plasma physics $[6,7,16,25,21,24,23,1,18]$. To obtain soliton solutions of these integrable systems is very difficult task for both mathematicians and physicists. In 1882, Darboux originated the idea about Darboux transformation when he was studying linear Sturm - Liouville problem. A century later in 1970, V. B Matveev extended his idea to important different partial differential equations (PDEs). Now a days Darboux transformation is very powerful tool to get soliton solutions and analyses it. A few authors studied different integrable models like nonlinear Schrodinger equation (NLS), 
Sine-Gordon equation and Korteweg-de Vries (KdV) equation and obtain soliton solutions by using Darboux transformation [27, 30, 17, 4, 5, 2].

The complex modified Korteweg-de Vries ( $\mathrm{mKdV}$ ) equation is very famous integrable model

$$
A_{t}+A_{x x x}+6|A|^{2} A_{x}=0
$$

This integrable system has been studied by number of researchers and obtained its breather solutions, conservation laws, periodic solutions, rogue wave solutions, M and $\mathrm{W}$ type solutions and interaction complex solitons $[26,8,10,9,20]$. Also, many researchers have been studied the generalizations of $\mathrm{mKdV}$ and obtained multi- soliton solutions. Vector version of $\mathrm{mKdV}$ was proposed in [15], solved initial value problem and obtain multi-soliton solutions in [19]. Iwao and Hirota [22] give coupled complex mKdV system as

$$
\begin{aligned}
& A_{t}+A_{x x x}+6\left(|A|^{2}+|B|^{2}\right) A_{x}=0, \\
& B_{t}+B_{x x x}+6\left(|A|^{2}+|B|^{2}\right) B_{x}=0 .
\end{aligned}
$$

This coupled (cmKdV) system used in orthogonally polarized transverse waves, molecular chain model and plasma waves $[3,28]$. When we study coupled $(\mathrm{cmKdV})$ to analyses the behavior of orthogonally polarized transverse waves, we find different geometrical orientation of oscillations. If we solve coupled $(\mathrm{cmKdV})$ system and find its exact solutions than we can easily compare and control the parameters of oscillations. Similar method canbe applied to molecular chain model and plasma waves. Different methods have been applied to different integrable systems to find different types of solutions [31,11]. Many authors obtained interesting kind of exact solutions called lump solutions [12, 29]. For constructing exact solutions of integrable nonlinear equations binary DT is very useful tool in soliton theory. In this method both spectral problem and corresponding adjoint associated with nonlinear system remain invariant by applying binary DT. Also the eigenvalues of Lax pair must be equal to the eigenvalues of its adjoint. In [13, 14], authors discuss the symmetries and reductions for the matrix $\mathrm{mKdV}$ and multicomponent NLS equations and find soliton solutions by using binary DT. In this article, we will use binary DT to obtain soliton solutions of system in equation (1.2). By iteration of binary DT along with quasideterminants, we will obtain multi- soliton solutions for both zero seed and nonzero seed solutions.

The sections of this article are divided as follows. In section 2, we briefly discuss the Lax pair of coupled(cmKdV) equation and standard binary DT. In section 3, many types of solutions are obtained for both zero seed and nonzero seed. The categories of these solutions are breather solutions, periodic solutions, rogue wave solutions, bright-dark solutions, bright-w-shaped solitons and bright-bright solitons.

\section{LAX PAIR AND BINARY DT}

2.1. Symmetries of Lax pair. The Lax pair of system in equation (1.2) is

$$
\begin{aligned}
& \Psi_{x}=-\left(P-\partial_{x}\right) \Psi, \\
& \Psi_{t}=-\left(Q-\partial_{t}\right) \Psi .
\end{aligned}
$$

Here

$$
P=\partial_{x}+\lambda J+R, \quad Q=\partial_{t}+4 \lambda^{3} J+4 \lambda^{2} R-2 \lambda M+W,
$$


With

$$
\begin{aligned}
J & =i\left(\begin{array}{cc}
I & O \\
O & -I
\end{array}\right), \quad R=\left(\begin{array}{cc}
O & -H \\
H^{\dagger} & O
\end{array}\right), \quad H=\left(\begin{array}{cc}
A^{*} & -B \\
B^{*} & A
\end{array}\right), \\
M & =i\left(\begin{array}{cc}
H H^{\dagger} & H_{x} \\
H_{x}^{\dagger} & -H H^{\dagger}
\end{array}\right), \quad W=\left(\begin{array}{cc}
H_{x} H^{\dagger}-H H_{x}^{\dagger} & H_{x x}+2 H H^{\dagger} H \\
-H_{x x}^{\dagger}-2 H^{\dagger} H H^{\dagger} & H_{x}^{\dagger} H-H^{\dagger} H_{x}
\end{array}\right) .
\end{aligned}
$$

Where $\dagger$ represent the Hermitian conjugate and $*$ denoted the complex conjugate. Also $I$ denoted $2 \times 2$ identity matrix and $O$ represent $2 \times 2$ zero matrix. The integrability condition $\Psi_{x t}=\Psi_{t x}$ give the system in equation (1.2).

Proposition 1. If $\eta=\left(\eta_{1}, \eta_{2}, \eta_{3}, \eta_{4}\right)^{T}$ is eigenfunction having eigenvalue $\lambda$, then $\zeta=$ $\left(\eta_{2}^{*},-\eta_{1}^{*}, \eta_{4}^{*},-\eta_{3}^{*}\right)^{T}$ is also eigenfunction of Lax pair in equation (2.3) having eigenvalue $-\lambda^{*}$.

So, we can write $4 \times 2$ matrix eigenfunction $\Lambda$ with $2 \times 2$ eigenvalue matrix $\theta$ as

$$
\Lambda=\left(\begin{array}{cc}
\eta_{1} & \eta_{2}^{*} \\
\eta_{2} & -\eta_{1}^{*} \\
\eta_{3} & \eta_{4}^{*} \\
\eta_{4} & -\eta_{3}^{*}
\end{array}\right), \quad \theta=\left(\begin{array}{cc}
\lambda & 0 \\
0 & -\lambda^{*}
\end{array}\right)
$$

Which are satisfying

$$
\Lambda_{x}+J \Lambda \theta+R \Lambda=0, \quad \Lambda_{t}+4 J \Lambda \theta^{3}-2 M \Lambda \theta+W \Lambda=0
$$

2.2. Binary Darboux Transformation. The linear differential operators are given as

$$
P=\partial_{x}+\sum_{i=0}^{N} A_{i} \partial_{y}^{i}, \quad Q=\partial_{t}+\sum_{i=0}^{N} B_{i} \partial_{y}^{i}
$$

Here $A_{i}$ and $B_{i}$ are $m \times m$ complex matrices.

The standard Darboux transformation can be presented as.

Theorem 1. The linear system is

$$
P(\eta)=Q(\eta)=0
$$

If $\Lambda$ be non-singular $m \times m$ matrix solution of equation (2.7).Then Darboux transformation $\widetilde{\eta}=H_{\Lambda}(\eta)=\Lambda \partial_{y} \Lambda^{-1} \eta$ leaves the system in equation (2.7) invariant.

$$
\widetilde{P}(\widetilde{\eta})=\widetilde{Q}(\widetilde{\eta})=0
$$

The operators $P$ and $Q$ have same values as $\widetilde{P}=H_{\Lambda} P H_{\Lambda}^{-1}$ and $\widetilde{Q}=H_{\Lambda} Q H_{\Lambda}^{-1}$ respectively. So,

$$
\widetilde{P}=\partial_{x}+\sum_{i=0}^{N} \widetilde{A}_{i} \partial_{y}^{i}, \quad \widetilde{Q}=\partial_{t}+\sum_{i=0}^{N} \widetilde{B}_{i} \partial_{y}^{i}
$$

By composing a DT with the inverse of another relative a binary DT is constructed as. 
Theorem 2. Let $\Lambda$ and $\sigma$ be $m \times k$ matrix solution of system in equation (2.7) and it adjoint $P^{\dagger}(\zeta)=Q^{\dagger}(\zeta)=0$ respectively. Then, a binary $D T$

$$
\begin{aligned}
& B_{\Lambda, \sigma}=I-\Lambda \Upsilon(\Lambda, \sigma)^{-1} \Upsilon(\cdot, \sigma) \\
& B_{\Lambda, \sigma}^{-\dagger}=I-\sigma \Upsilon(\Lambda, \sigma)^{-\dagger} \Upsilon(\Lambda, \cdot)^{\dagger}
\end{aligned}
$$

Here $\Upsilon(\Lambda, \sigma)_{y}=\sigma^{\dagger} \Lambda$ preserve the system in equation (2.7) and it adjoint $P^{\dagger}(\zeta)=$ $Q^{\dagger}(\zeta)=0$ respectively. Nth-iterated binary DT is constructed as.

Theorem 3. Let $\Lambda_{1}, \Lambda_{2}, \ldots, \Lambda_{N}$ be $N$ linearly independent solutions of system in equation (2.7) and $\sigma_{1}, \sigma_{2}, \ldots ., \sigma_{N}$ be $N$ linearly independent solution of it adjoint $P^{\dagger}(\zeta)=Q^{\dagger}(\zeta)=$

0 . Then $N$-fold binary DT can be denoted in the term of quasideterminants as

$$
\begin{aligned}
& \eta[N]=\left|\begin{array}{cc}
\Upsilon(\Theta, \Sigma) & \Upsilon(\eta, \Sigma) \\
\Theta & \eta
\end{array}\right|, \\
& \zeta[N]=\left|\begin{array}{cc}
\Upsilon(\Theta, \Sigma)^{\dagger} & \Upsilon(\Theta, \zeta)^{\dagger} \\
\Sigma & \zeta
\end{array}\right| .
\end{aligned}
$$

Where $\Theta=\left(\Lambda_{1}, \Lambda_{2}, \ldots, \Lambda_{N}\right), \Sigma=\left(\sigma_{1}, \sigma_{2}, \ldots, \sigma_{N}\right), \Upsilon(\Theta, \Sigma)=\Upsilon\left(\left(\Lambda_{i}, \sigma_{i}\right)\right)_{i j=1,2, \ldots N}$ is $N \times N$ matrix, $\Upsilon(\eta, \Sigma)=\left(\Upsilon\left(\eta, \sigma_{j}\right)\right)_{j=1,2, \ldots, N}$ is $N \times 1$ vector and $\Upsilon(\Theta, \zeta)=$ $\left(\Upsilon\left(\zeta, \Lambda_{i}\right)\right)_{i=1,2, \ldots N}$ is a $1 \times N$ vector.

By using separation of variables technique we reduce binary DT from $(2+1)$ to $(1+1)$ dimensions.

$$
\begin{aligned}
\eta=\eta^{d}(x, t) e^{\lambda y}, & \Lambda=\Lambda^{d}(x, t) e^{\theta y}, \\
\zeta=\zeta^{d}(x, t) e^{\mu y}, & \sigma=\sigma^{d}(x, t) e^{\Pi y}
\end{aligned}
$$

Where $\theta, \Pi$ are $N \times N$ constant matrices and $\lambda, \mu$ are constant scalars. So, matrix operators $P$ and $Q$ becomes

$$
P^{d}=\partial_{x}+\sum_{i=0}^{N} A_{i} \lambda^{i}, \quad Q^{d}=\partial_{t}+\sum_{i=0}^{N} B_{i} \lambda^{i}
$$

From above $y$ dependence potential $\Upsilon$ write explicitly as

$$
\Upsilon(\Lambda, \sigma)=e^{\Pi^{\dagger} y} \Upsilon^{d}\left(\Lambda^{d}, \sigma^{d}\right) e^{\theta y}, \quad \Upsilon(\eta, \sigma)=e^{\left(\Pi^{\dagger}+\lambda I\right) y} \Upsilon^{d}\left(\eta^{d}, \sigma^{d}\right),
$$

$\Pi$ and $\theta$ satisfy

$$
\Pi^{\dagger} \Upsilon^{d}\left(\Lambda^{d}, \sigma^{d}\right)+\Upsilon^{d}\left(\Lambda^{d}, \sigma^{d}\right)=\sigma^{d \dagger} \Lambda^{d}, \quad\left(\Pi^{\dagger}+\lambda I\right) \Upsilon^{d}\left(\eta^{d}, \sigma^{d}\right)=\sigma^{d \dagger} \eta^{d}
$$

We discuss reduced case and ignore superscript $d$. If the constant matrices to be diagonal $\Pi=\operatorname{diag}\left(\mu_{1}, \mu_{2}, \ldots ., \mu_{N}\right)$ and $\Theta=\operatorname{diag}\left(\lambda_{1}, \lambda_{2}, \ldots ., \lambda_{N}\right)$ then we obtain as

$$
\Upsilon(\Lambda, \sigma)=\frac{\left(\sigma^{\dagger} \Lambda\right)_{i j}}{\lambda_{j}+\mu_{i}^{*}}, \quad \Upsilon(\eta, \sigma)=\frac{\left(\sigma^{\dagger} \eta\right)_{i j}}{\lambda_{j}+\mu_{i}^{*}}, \quad(i, j=1,2, \ldots, N)
$$

As $J$ and $R$ are skew-Hermitian, then by using property $P+P^{\dagger}=0, Q+Q^{\dagger}=0$ keep constraints among potentials in $R$, we choose $\sigma=\Lambda$ and $\Pi=-\theta$ and also $P$ is invariant under binary DT

$$
P \rightarrow \widetilde{P}=B_{\Lambda, \sigma} P B_{\Lambda, \sigma}^{-1}=\partial_{x}+\lambda J+\widetilde{R}
$$


Here

$$
\widetilde{R}=R+\left[J, \Lambda \Upsilon(\Lambda, \Lambda)^{-1} \Lambda^{\dagger}\right]
$$

For simplicity, we introduce $4 \times 4$ matrix

$$
S=\frac{1}{2 i}\left(\begin{array}{cc}
O & H \\
H^{\dagger} & O
\end{array}\right)
$$

and $R=[S, J]$ then

$$
\widetilde{S}=S+\left|\begin{array}{cc}
\Upsilon(\Lambda, \Lambda) & \Lambda^{\dagger} \\
\Lambda & \mathrm{O}
\end{array}\right|=S-\Lambda \Upsilon(\Lambda, \Lambda)^{-1} \Lambda^{\dagger}
$$

Here $\Upsilon(\Lambda, \Lambda)$ satisfy property

$$
\Upsilon(\Lambda, \Lambda) \theta-\Lambda^{\dagger} \Upsilon(\Lambda, \Lambda)=\Lambda^{\dagger} \Lambda
$$

Theorem 4. Let $\Lambda_{1}, \Lambda_{2}, \ldots, \Lambda_{N}$ are $N$ different linearly independent solutions of system $P(\eta)=Q(\eta)=0$ with $\lambda_{1}, \lambda_{2}, \ldots, \lambda_{N}$. Then $N$-fold iterative potential transformation becomes

$$
S[N]=S+\left|\begin{array}{cc}
\Upsilon(\Theta, \Theta) & \Theta^{\dagger} \\
\Theta & O
\end{array}\right|
$$

Where

$$
\Upsilon(\Theta, \Theta)=\left(\begin{array}{cccccc}
\Upsilon\left(\Lambda_{1}, \Lambda_{1}\right) & \Upsilon\left(\Lambda_{2}, \Lambda_{1}\right) & \cdot & \cdot & \cdot & \Upsilon\left(\Lambda_{N}, \Lambda_{1}\right) \\
\Upsilon\left(\Lambda_{1}, \Lambda_{2}\right) & \Upsilon\left(\Lambda_{2}, \Lambda_{2}\right) & \cdot & \cdot & \cdot & \Upsilon\left(\Lambda_{N}, \Lambda_{2}\right) \\
\cdot & \cdot & \cdot & & & \cdot \\
\cdot & \cdot & & \cdot & & \cdot \\
\cdot & \cdot & & & \cdot & \cdot \\
\Upsilon\left(\Lambda_{1}, \Lambda_{N}\right) & \Upsilon\left(\Lambda_{2}, \Lambda_{N}\right) & \cdot & \cdot & \cdot & \Upsilon\left(\Lambda_{N}, \Lambda_{N}\right)
\end{array}\right)
$$

With

$$
\Theta=\left(\Lambda_{1}, \Lambda_{2}, \ldots, \Lambda_{N}\right), \Lambda_{k}=\left(\begin{array}{cc}
\eta_{4 k-3} & \eta_{4 k-2}^{*} \\
\eta_{4 k-2} & -\eta_{4 k-3}^{*} \\
\eta_{4 k-1} & \eta_{4 k}^{*} \\
\eta_{4 k} & -\eta_{4 k-1}^{*}
\end{array}\right) \quad, \quad k=(1,2, \ldots, N)
$$

Here $\Upsilon\left(\Lambda_{i}, \Lambda_{j}\right)$ satisfy

$$
\Upsilon\left(\Lambda_{i}, \Lambda_{j}\right) \theta_{i}-\theta_{j}^{\dagger} \Upsilon\left(\Lambda_{i}, \Lambda_{j}\right)=\Lambda_{j}^{\dagger} \Lambda_{i}, \quad \theta_{i}=\operatorname{diag}\left(\lambda_{i},-\lambda_{i}^{*}\right), \quad(i, j=1,2, \ldots, N)
$$

Potential $\Upsilon$ can be written explicitly from above

$$
\Upsilon\left(\Lambda_{i}, \Lambda_{j}\right)=\left(\begin{array}{cc}
F_{i j} & H_{i j}^{*} \\
H_{i j} & -F_{i j}
\end{array}\right)
$$

Where

$$
\begin{aligned}
F_{i j} & =\frac{1}{\lambda_{i}-\lambda_{j}^{*}}\left(\eta_{4 i} \eta_{4 j}^{*}+\eta_{4 i-1} \eta_{4 j-1}^{*}+\eta_{4 i-2} \eta_{4 j-2}^{*}+\eta_{4 i-3} \eta_{4 j-3}^{*}\right) \\
G_{i j} & =\frac{1}{\lambda_{i}-\lambda_{j}}\left(\eta_{4 i-3} \eta_{4 j-2}-\eta_{4 i-2} \eta_{4 j-3}+\eta_{4 i-1} \eta_{4 j}+\eta_{4 i} \eta_{4 j-1}\right) .
\end{aligned}
$$


Inserting eq. (2.24) into eq. (2.22) we get

$$
\begin{aligned}
A[N] & =A+2 i\left|\begin{array}{cc}
\Upsilon(\Theta, \Theta) & \Psi_{1}^{\dagger} \\
\Psi_{3} & \mathrm{O}
\end{array}\right|=A+2 i\left|\begin{array}{cc}
\Upsilon(\Theta, \Theta) & \Psi_{4}^{\dagger} \\
\Psi_{2} & \mathrm{O}
\end{array}\right|, \\
B[N] & =B+2 i\left|\begin{array}{cc}
\Upsilon(\Theta, \Theta) & \Psi_{2}^{\dagger} \\
\Psi_{3} & \mathrm{O}
\end{array}\right|=B-2 i\left|\begin{array}{cc}
\Upsilon(\Theta, \Theta) & \Psi_{4}^{\dagger} \\
\Psi_{1} & \mathrm{O}
\end{array}\right|, \\
A[N]^{*} & =A^{*}+2 i\left|\begin{array}{cc}
\Upsilon(\Theta, \Theta) & \Psi_{3}^{\dagger} \\
\Psi_{1} & \mathrm{O}
\end{array}\right|=A^{*}+2 i\left|\begin{array}{cc}
\Upsilon(\Theta, \Theta) & \Psi_{2}^{\dagger} \\
\Psi_{4} & \mathrm{O}
\end{array}\right|, \\
B[N]^{*} & =B^{*}+2 i\left|\begin{array}{cc}
\Upsilon(\Theta, \Theta) & \Psi_{3}^{\dagger} \\
\Psi_{2} & \mathrm{O}
\end{array}\right|=B^{*}-2 i\left|\begin{array}{cc}
\Upsilon(\Theta, \Theta) & \Psi_{1}^{\dagger} \\
\Psi_{4} & \mathrm{O}
\end{array}\right| .
\end{aligned}
$$

Here $\Psi_{j}=(j=1,2,3,4)$ is $j$ th row of matrix $\Theta$. We can easily check these relation by applying quasideterminants properties.

\section{ZERO SEED AND NON-ZERO SEED SOLUTIONS OF COUPLED (CMKDV) SYSTEM}

We construct different exact solutions in this section for both zero seed and non-zero seed of coupled (cmKdV) system.

3.1. Zero seed solutions. By using quasideterminants properties to the transformations in equation (2.26) one can easily obtain once-iterated transformations as

$$
\begin{aligned}
& {[A]=A-2 i \frac{\left(\lambda_{1}-\lambda_{1}^{*}\right)\left(\eta_{1}^{*} \eta_{3}+\eta_{2} \eta_{4}^{*}\right)}{\left|\eta_{1}\right|^{2}+\left|\eta_{2}\right|^{2}+\left|\eta_{3}\right|^{2}+\left|\eta_{4}\right|^{2}},} \\
& {[B]=B-2 i \frac{\left(\lambda_{1}-\lambda_{1}^{*}\right)\left(\eta_{2}^{*} \eta_{3}+\eta_{1} \eta_{4}^{*}\right)}{\left|\eta_{1}\right|^{2}+\left|\eta_{2}\right|^{2}+\left|\eta_{3}\right|^{2}+\left|\eta_{4}\right|^{2}} .}
\end{aligned}
$$

The system in equation (2.7) become when $A=B=0$ of equation (1.2) for zero seed solutions.

$$
\begin{aligned}
\eta_{x}+\lambda J \eta & =0 \\
\eta_{t}+4 \lambda^{3} J \eta & =0 .
\end{aligned}
$$

having solution

$$
\eta=\left(\alpha e^{-i \rho}, \beta e^{-i \rho}, \gamma e^{i \rho}, \delta e^{i \rho}\right)^{T}, \quad \rho=\lambda\left(x+4 \lambda^{2} t\right)
$$

3.1.1. Breather solution. When $N=1$, by substituting solution $\eta^{[1]}=\left(\eta_{1}, \eta_{2}, \eta_{3}, \eta_{4}\right)^{T}=$ $\left(\alpha_{1} e^{-i \rho_{1}}, \beta_{1} e^{-i \rho_{1}}, \gamma_{1} e^{i \rho_{1}}, \delta_{1} e^{i \rho_{1}}\right)^{T}$ with $\rho_{1}=\lambda_{1}\left(x+4 \lambda^{2} t\right)$ where $\lambda_{1}=a_{1}+i b_{1},\left(b_{1} \neq 0\right)$ 
in equation (3.27) one can get breather solution of equation (1.2) as

$$
\begin{aligned}
A[1]= & \frac{4 b_{1}\left[\left(\alpha_{1}^{*} \gamma_{1}+\beta_{1} \delta_{1}^{*}\right) \cos \left(-2 a_{1}\left[x+4\left(a_{1}^{2}-3 b_{1}^{2}\right) t\right]\right)\right.}{\left(\left|\alpha_{1}\right|^{2}+\left|\beta_{1}\right|^{2}+\left|\gamma_{1}\right|^{2}+\left|\delta_{1}\right|^{2}\right) \cosh \left(-2 b_{1}\left[x+4\left(3 a_{1}^{2}-b_{1}^{2}\right) t\right]\right)} \\
& \frac{\left.+i\left(\beta_{1} \delta_{1}^{*}-\alpha_{1}^{*} \gamma_{1}\right) \sin \left(-2 a_{1}\left[x+4\left(a_{1}^{2}-3 b_{1}^{2}\right) t\right]\right)\right]}{+\left(\left|\alpha_{1}\right|^{2}+\left|\beta_{1}\right|^{2}+\left|\gamma_{1}\right|^{2}+\left|\delta_{1}\right|^{2}\right) \sinh \left(-2 b_{1}\left[x+4\left(3 a_{1}^{2}-b_{1}^{2}\right) t\right]\right)}, \\
B[1]= & \frac{4 b_{1}\left[\left(\alpha_{1} \delta_{1}^{*}-\beta_{1}^{*} \gamma_{1}\right) \cos \left(-2 a_{1}\left[x+4\left(a_{1}^{2}-3 b_{1}^{2}\right) t\right]\right)\right.}{\left(\left|\alpha_{1}\right|^{2}+\left|\beta_{1}\right|^{2}+\left|\gamma_{1}\right|^{2}+\left|\delta_{1}\right|^{2}\right) \cosh \left(-2 b_{1}\left[x+4\left(3 a_{1}^{2}-b_{1}^{2}\right) t\right]\right)} \\
& \frac{\left.+i\left(\alpha_{1} \delta_{1}^{*}+\beta_{1}^{*} \gamma_{1}\right) \sin \left(-2 a_{1}\left[x+4\left(a_{1}^{2}-3 b_{1}^{2}\right) t\right]\right)\right]}{+\left(\left|\alpha_{1}\right|^{2}+\left|\beta_{1}\right|^{2}+\left|\gamma_{1}\right|^{2}+\left|\delta_{1}\right|^{2}\right) \sinh \left(-2 b_{1}\left[x+4\left(3 a_{1}^{2}-b_{1}^{2}\right) t\right]\right)} .
\end{aligned}
$$

For simplicity we use notations as

$$
\begin{aligned}
v & =-2 a_{1}\left[x+4\left(a_{1}^{2}-3 b_{1}^{2}\right) t\right], \quad \varsigma=-2 b_{1}\left[x+4\left(3 a_{1}^{2}-b_{1}^{2}\right) t\right], \\
l_{1} & =\left|\alpha_{1}\right|^{2}+\left|\beta_{1}\right|^{2}+\left|\gamma_{1}\right|^{2}+\left|\delta_{1}\right|^{2}, \quad l_{2}=\left(\left|\gamma_{1}\right|^{2}+\left|\delta_{1}\right|^{2}-\left|\alpha_{1}\right|^{2}-\left|\beta_{1}\right|^{2}\right)(3
\end{aligned}
$$

Now the solution in equation (3.30) changed to

$$
\begin{aligned}
& A[1]=\frac{4 b_{1}\left[\left(\alpha_{1}^{*} \gamma_{1}+\beta_{1} \delta_{1}^{*}\right) \cos (v)+i\left(\beta_{1} \delta_{1}^{*}-\alpha_{1}^{*} \gamma_{1}\right) \sin (v)\right]}{l_{1} \cosh (\varsigma)+l_{2} \sinh (\varsigma)} \\
& B[1]=\frac{4 b_{1}\left[\left(\alpha_{1} \delta_{1}^{*}-\beta_{1}^{*} \gamma_{1}\right) \cos (v)+i\left(\alpha_{1} \delta_{1}^{*}+\beta_{1}^{*} \gamma_{1}\right) \sin (v)\right]}{l_{1} \cosh (\varsigma)+l_{2} \sinh (\varsigma)}
\end{aligned}
$$
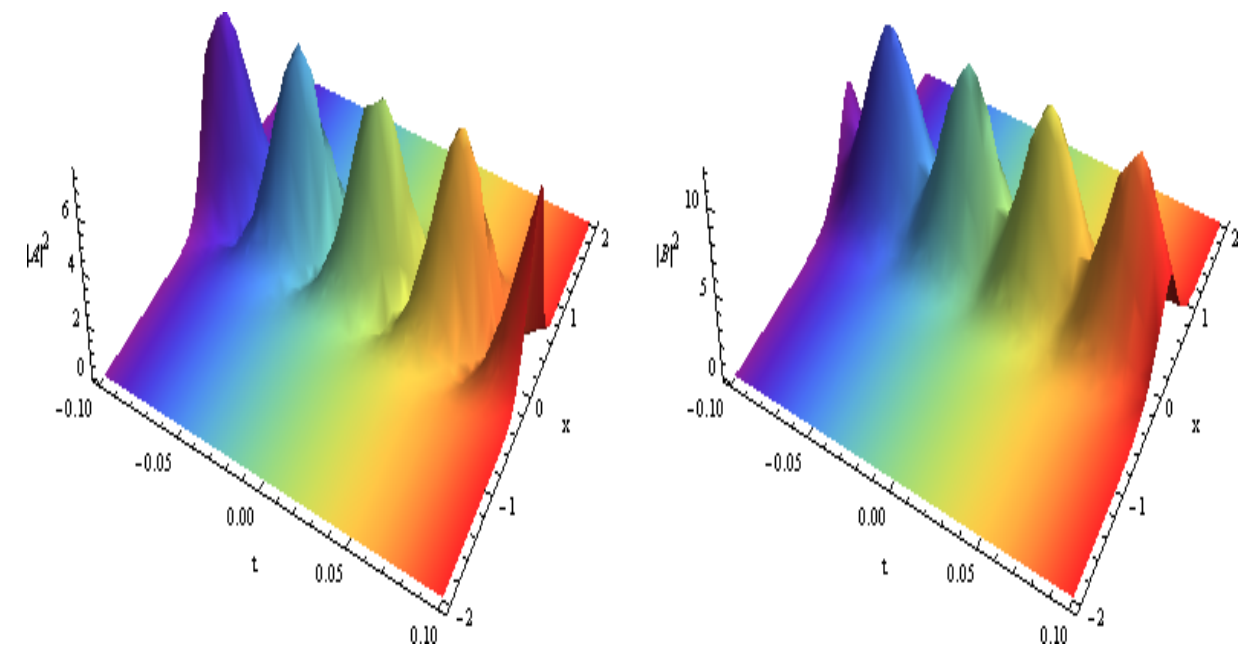

Figure:1 Breather solutions having parameters: $\alpha_{1}=\frac{1}{2}, \beta_{1}=\delta_{1}=1, \gamma_{1}=2, \lambda_{1}=$ $\sqrt{3} i+1$

3.1.2. Bright one-soliton solutions. The breather solutions in equation (3.32) are defined by parameters $a_{1}, b_{1}, \alpha_{1}, \beta_{1}, \gamma_{1}$ and $\delta_{1}$. If we take zero any one of these then we obtain 
bright one-soliton solution. we take $\alpha_{1}=0$ to get the following solutions as

$$
\begin{aligned}
& A[1]=\frac{\left(4 b_{1} \beta_{1} \delta_{1}^{*}\right) e^{i v}}{\left|\beta_{1}\right|^{2}+\left|\gamma_{1}\right|^{2}+\left|\delta_{1}\right|^{2} \cosh (\varsigma)\left|\gamma_{1}\right|^{2}+\left|\delta_{1}\right|^{2}-\left|\beta_{1}\right|^{2} \sinh (\varsigma)}, \\
& B[1]=\frac{\left(4 b_{1} \beta_{1}^{*} \gamma_{1}\right) e^{-i v}}{\left|\beta_{1}\right|^{2}+\left|\gamma_{1}\right|^{2}+\left|\delta_{1}\right|^{2} \cosh (\varsigma)\left|\gamma_{1}\right|^{2}+\left|\delta_{1}\right|^{2}-\left|\beta_{1}\right|^{2} \sinh (\varsigma)} .
\end{aligned}
$$

If

$$
l_{3}=\left|\beta_{1}\right|^{2}+\left|\gamma_{1}\right|^{2}+\left|\delta_{1}\right|^{2}, \quad l_{4}=\left|\gamma_{1}\right|^{2}+\left|\delta_{1}\right|^{2}-\left|\beta_{1}\right|^{2}
$$

Then the solutions becomes

$$
\begin{aligned}
A[1] & =\frac{\left(4 b_{1} \beta_{1} \delta_{1}^{*}\right) e^{i v}}{l_{3} \cosh (\varsigma) l_{4} \sinh (\varsigma)} \\
B[1] & =\frac{\left(4 b_{1} \beta_{1}^{*} \gamma_{1}\right) e^{-i v}}{l_{3} \cosh (\varsigma) l_{4} \sinh (\varsigma)} .
\end{aligned}
$$
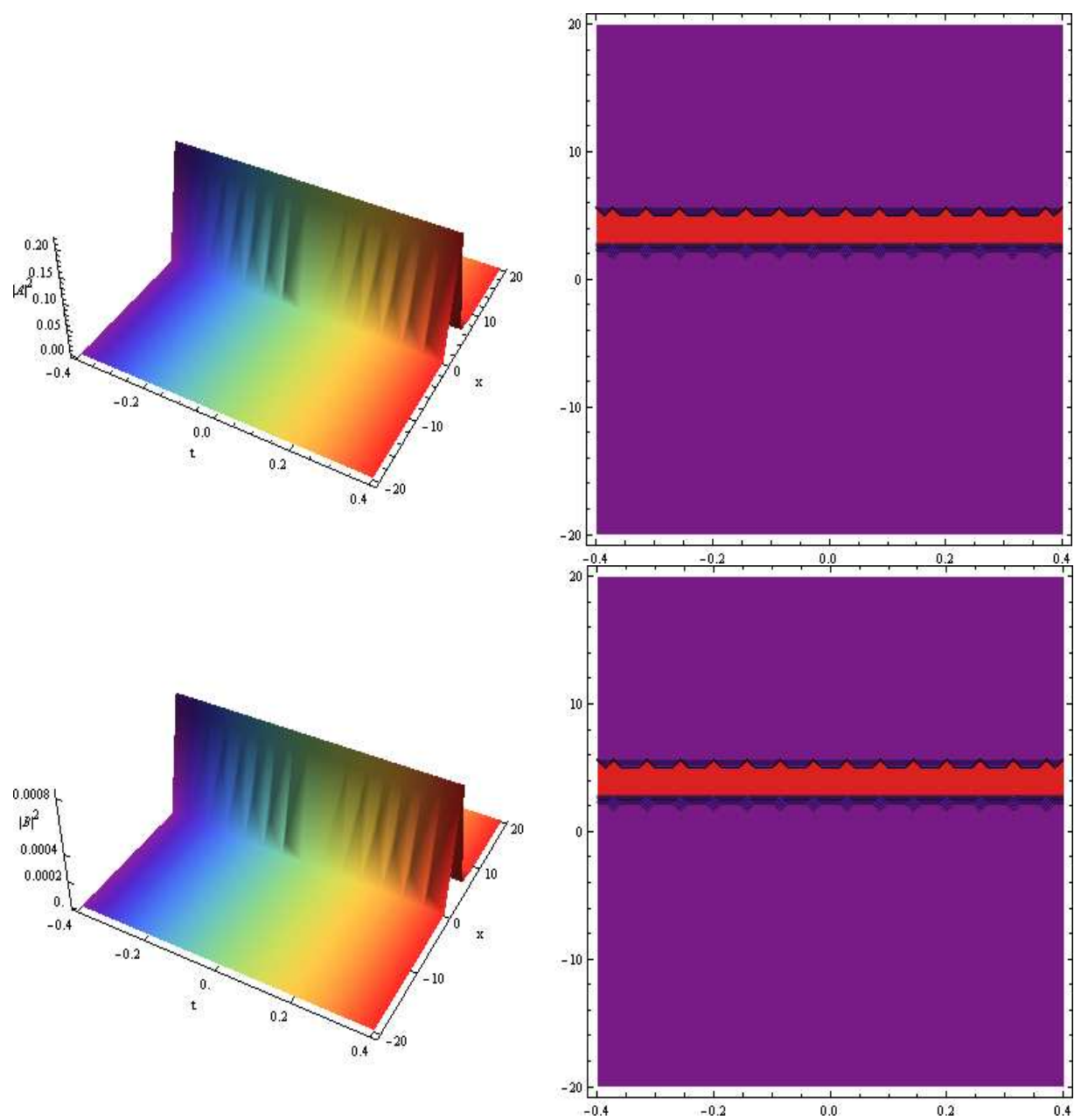
Figure:2 Bright one-soliton with parameters: $\alpha_{1}=2, \beta_{1}=1, \gamma_{1}=\frac{1}{2}, \delta_{1}=0$ and $\lambda_{1}=i+\frac{1}{8}$

By using same algorithm we can easily obtain two-soliton solution when $N=2$.

\subsection{Non-zero seed solutions.}

3.2.1. Case 1. Let $g_{j}(j=1,2)$ is a complex constants for non-zero seed solution of $A$ and $B$. Inserting $A$ and $B$ into equation (2.7) we get the solution as $\eta=\left(\eta_{1}, \eta_{2}, \eta_{3}, \eta_{4}\right)^{T}$, where

$$
\begin{aligned}
& \eta_{1}=\beta e^{\vartheta}+\gamma e^{-\vartheta}, \\
& \eta_{2}=\delta e^{\vartheta}+\alpha e^{-\vartheta}, \\
& \eta_{3}=e^{\vartheta} \varrho^{+}\left(g_{2} \delta+g_{1} \beta\right)+e^{-\vartheta} \varrho^{-}\left(g_{2} \alpha+g_{1} \gamma\right), \\
& \eta_{4}=e^{\vartheta} \varrho^{+}\left(g_{1}^{*} \delta+g_{2}^{*} \beta\right)+e^{-\vartheta} \varrho^{-}\left(g_{1}^{*} \alpha+g_{2}^{*} \gamma\right) .
\end{aligned}
$$

Where

$$
\begin{aligned}
\vartheta & =i \sqrt{\left|g_{1}\right|^{2}+\left|g_{2}\right|^{2}+\lambda^{2}}\left[x+2\left(2 \lambda^{2}-\left(\left|g_{1}\right|^{2}+\left|g_{2}\right|^{2}\right) t\right]\right. \\
\varrho^{ \pm} & =\frac{i \lambda \pm i \sqrt{\lambda^{2}+\left|g_{1}\right|^{2}+\left|g_{2}\right|^{2}}}{\left|g_{1}\right|^{2}+\left|g_{2}\right|^{2}} .
\end{aligned}
$$

We can get the solutions of equation (1.2) by substituting equation (3.36-3.39) into equation (3.27). Here we choose $\lambda_{1}=i h(h \neq 0)$. This leads to two different cases for parameters values.

(1). If $h>\sqrt{\left|g_{1}\right|^{2}+\left|g_{2}\right|^{2}}$, then the terms in equation (3.40) changed to

$$
\begin{aligned}
\vartheta & =-\sqrt{h^{2}-\left(\left|g_{1}\right|^{2}+\left|g_{2}\right|^{2}\right)}\left[x-2\left(2 h^{2}+\left(\left|g_{1}\right|^{2}+\left|g_{2}\right|^{2}\right) t\right],\right. \\
\varrho^{ \pm} & =\frac{-h \mp \sqrt{h^{2}-\left(\left|g_{1}\right|^{2}+\left|g_{2}\right|^{2}\right)}}{\left|g_{1}\right|^{2}+\left|g_{2}\right|^{2}} .
\end{aligned}
$$

Upon calculation, we obtain

$$
\begin{aligned}
& A[1]=g_{1}\left(1-\frac{4 h E_{1}}{g_{1} k}\right), \\
& B[1]=g_{2}\left(1-\frac{4 h E_{2}}{g_{2} k}\right) .
\end{aligned}
$$


Where

$$
\begin{aligned}
E_{1}= & -e^{2 \vartheta} \varrho^{+} g_{1}\left(|\beta|^{2}+|\delta|^{2}\right)-e^{-2 \vartheta} \varrho^{-} g_{1}\left(|\alpha|^{2}+|\gamma|^{2}\right) \\
& -\varrho^{+}\left[g_{1}\left(\beta \gamma^{*}+\delta \alpha^{*}\right)+g_{2}\left(\delta \gamma^{*}-\beta^{*} \alpha\right)\right] \\
& -\varrho^{-}\left[g_{1}\left(\gamma \beta^{*}+\alpha^{*} \delta\right)+g_{2}\left(\alpha \beta^{*}-\gamma^{*} \delta\right)\right], \\
E_{2}= & -e^{2 \vartheta} \varrho^{+} g_{2}\left(|\beta|^{2}+|\delta|^{2}\right)-e^{-2 \vartheta} \varrho^{-} g_{2}\left(|\alpha|^{2}+|\gamma|^{2}\right) \\
& -\varrho^{+}\left[g_{1}\left(\beta \alpha^{*}+\delta^{*} \gamma\right)+g_{2}\left(\delta \alpha^{*}-\beta^{*} \gamma\right)\right] \\
& -\varrho^{-}\left[g_{1}\left(\gamma \delta^{*}+\alpha^{*} \beta\right)+g_{2}\left(\alpha \delta^{*}-\gamma^{*} \beta\right)\right], \\
k= & e^{2 \vartheta}\left(|\beta|^{2}+|\delta|^{2}\right)\left[1+\varrho^{+} \varrho^{+}\left(\left|g_{1}\right|^{2}+\left|g_{2}\right|^{2}\right)\right] \\
& +e^{-2 \vartheta}\left(|\gamma|^{2}+|\alpha|^{2}\right)\left[1+\varrho^{-} \varrho^{-}\left(\left|g_{1}\right|^{2}+\left|g_{2}\right|^{2}\right)\right] \\
& +\left(\beta \gamma^{*}+\beta^{*} \gamma+\delta \alpha^{*}+\delta^{*} \alpha\right)\left[1+\varrho^{+} \varrho^{-}\left(\left|g_{1}\right|^{2}+\left|g_{2}\right|^{2}\right)\right] .
\end{aligned}
$$

By choosing suitable parameters we obtain three kinds of solution of equation (3.42-3.43)

Bright-bright soliton solution: If the parameters are $\alpha=\beta=\gamma=\delta=g_{1}=1, g_{2}=$ $-i$ and $h=2$ then both equation (3.42-3.43) give bright-bright solitons.
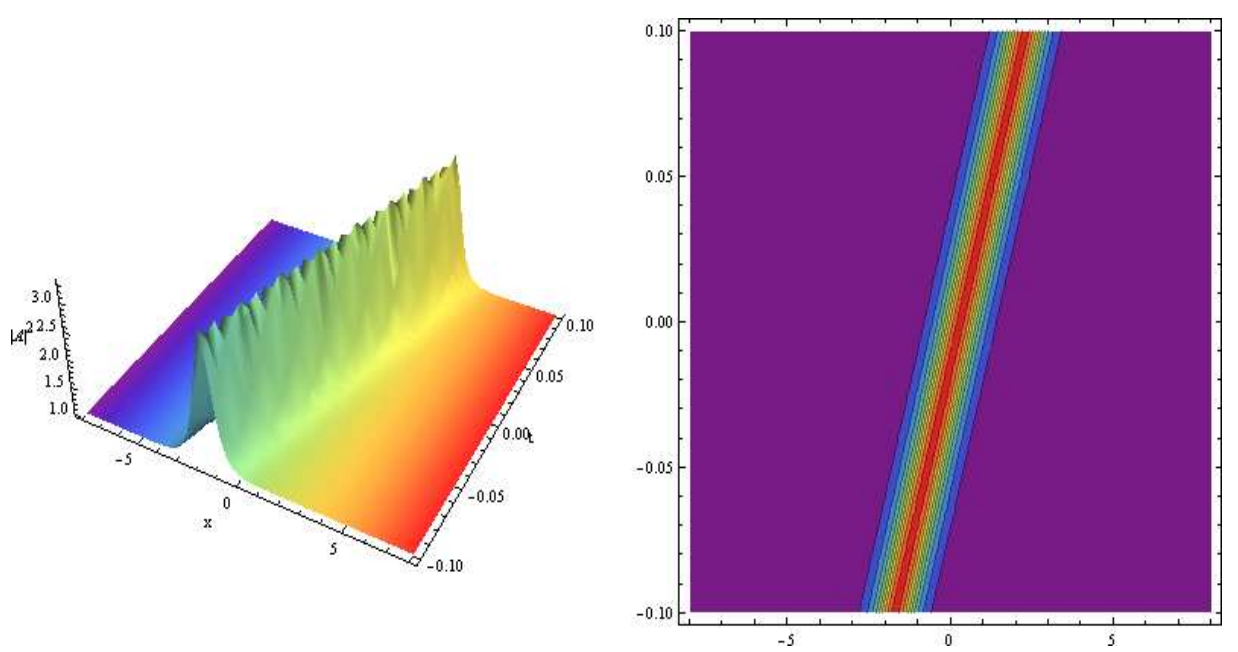

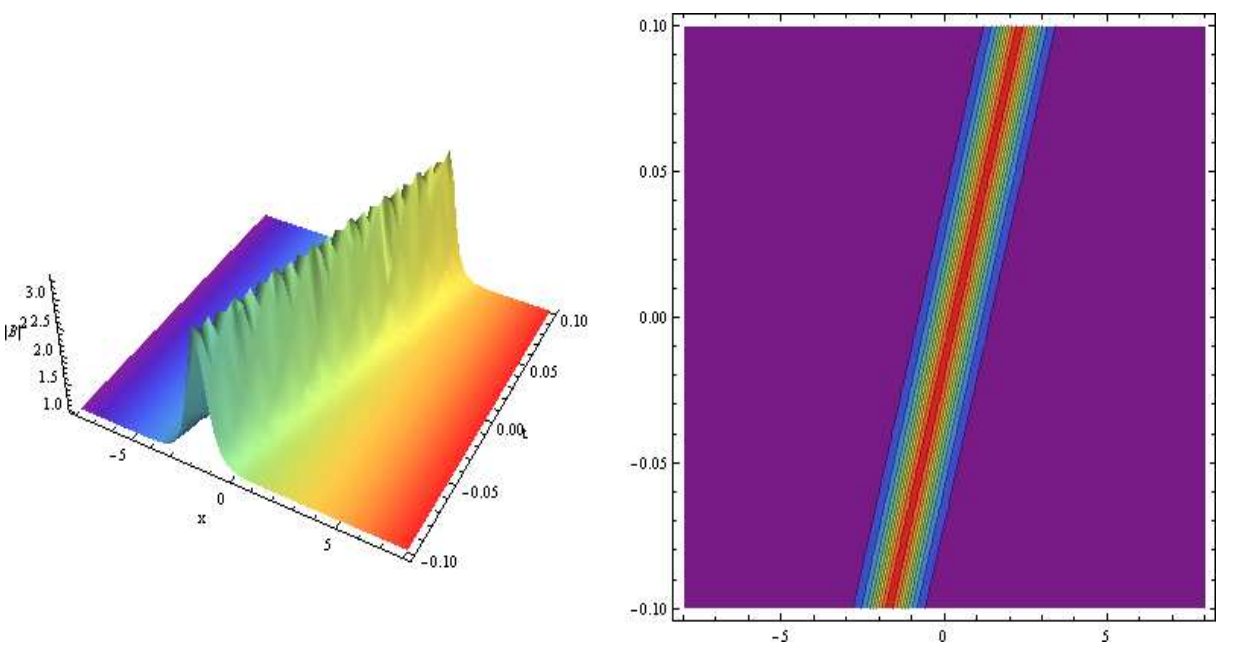

Figure:3 Bright-bright solitons having parameters $\alpha=\beta=\gamma=\delta=g_{1}=1, g_{2}=$ $-i, h=2$

Bright-dark soliton solution: If the parameters are $\alpha=\gamma=\delta=g_{1}=1, \beta=0, g_{2}=$ -1 and $h=2$ then solution equation (3.42) is dark and equation (3.43) is bright.
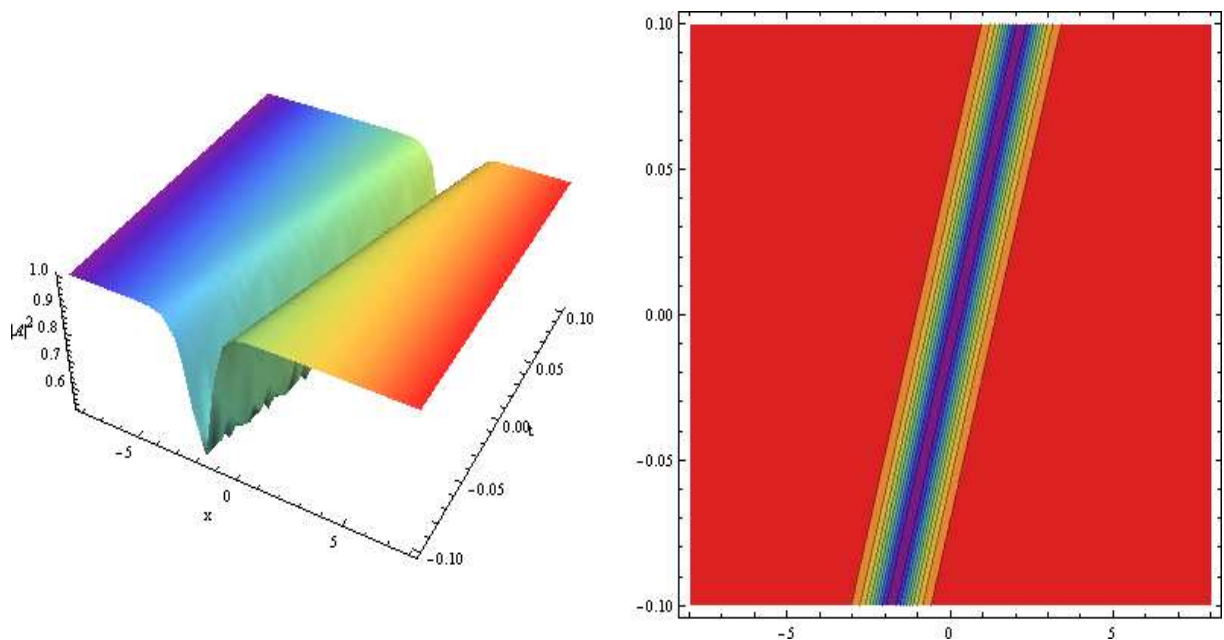

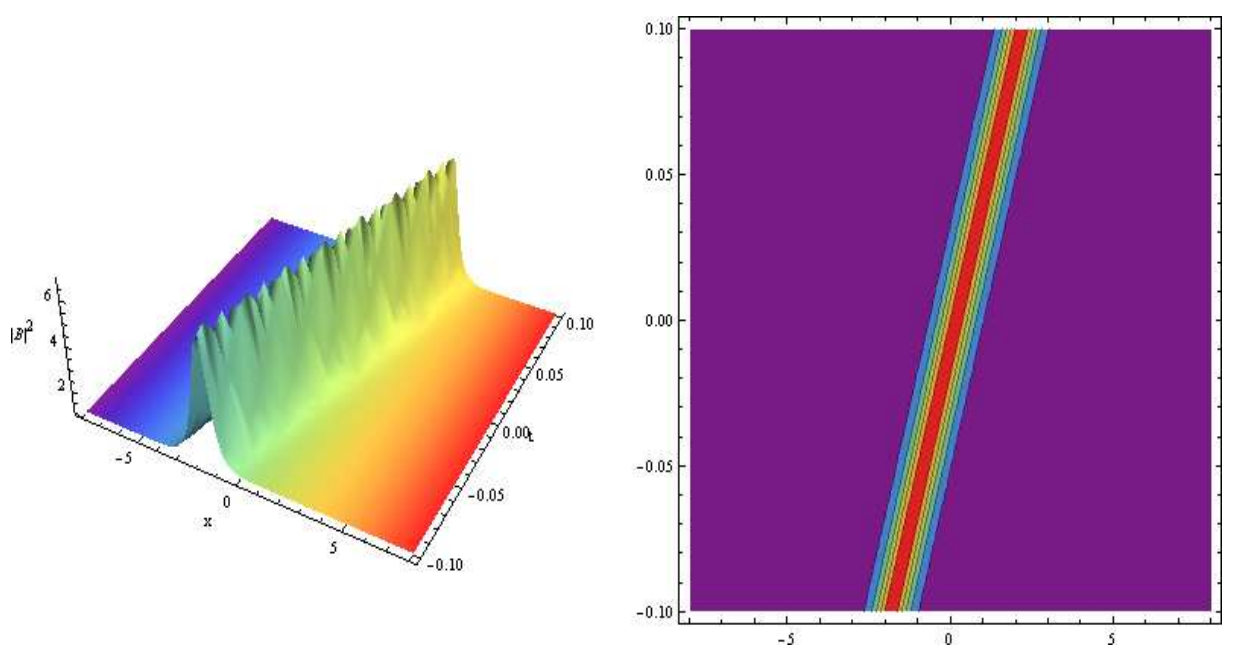

Figure:4 Bright-dark solitons having parameters $\alpha=\gamma=\delta=g_{1}=1, \beta=0, g_{2}=$ $-1, h=2$

W-shaped soliton solution: If the parameters are $\alpha=\beta=\delta=g_{1}=1, g_{2}=2, h=$ $\sqrt{15}$ then the solution in equation (3.42) is w-shaped and (3.43) is bright solitons.
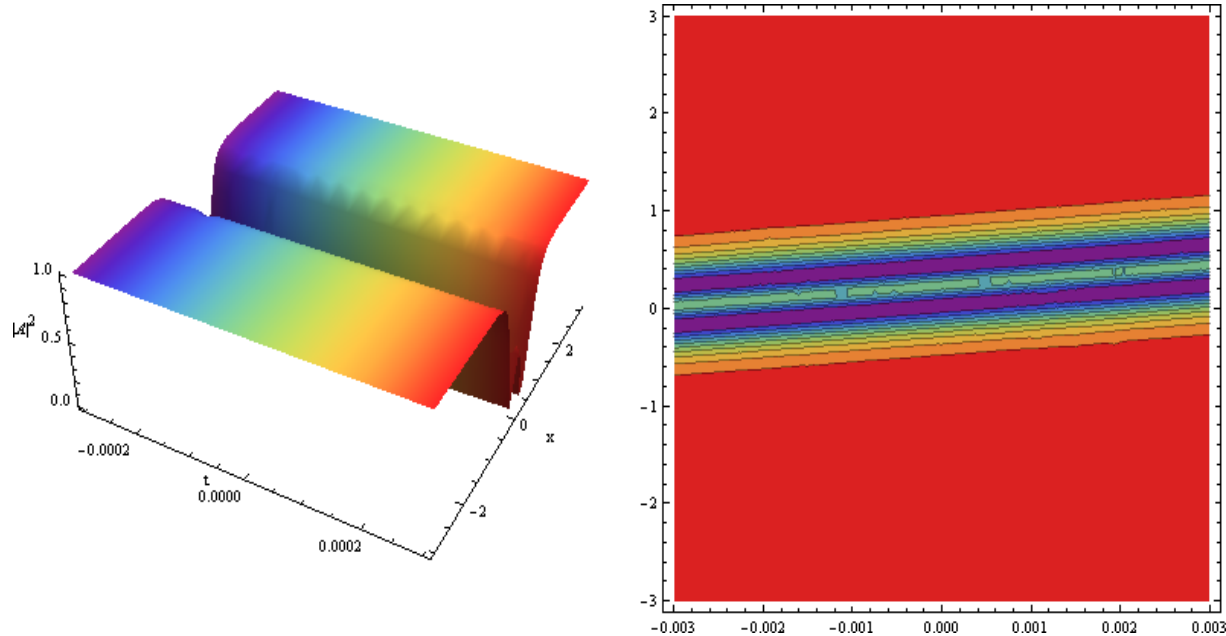

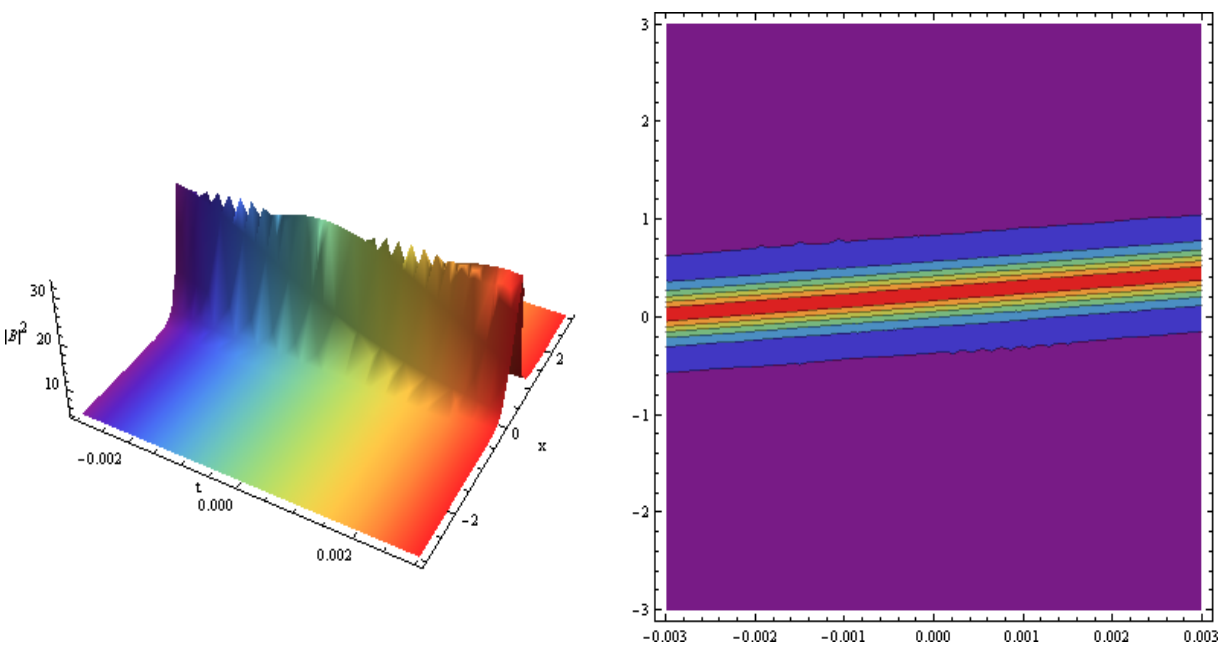

Figure:5 having parameters $\alpha=\beta=\delta=g_{1}=1, g_{2}=2, h=\sqrt{15}$

(2) If $h<\sqrt{\left|g_{1}\right|^{2}+\left|g_{2}\right|^{2}}$, then the terms in equation (3.40) changed to

$$
\begin{aligned}
\vartheta & =i \sqrt{\left(\left|g_{1}\right|^{2}+\left|g_{2}\right|^{2}\right)-h^{2}}\left[x-2\left(2 h^{2}+\left(\left|g_{1}\right|^{2}+\left|g_{2}\right|^{2}\right) t\right],\right. \\
\varrho^{ \pm} & =\frac{-h \pm i \sqrt{\left(\left|g_{1}\right|^{2}+\left|g_{2}\right|^{2}\right)-h^{2}}}{\left|g_{1}\right|^{2}+\left|g_{2}\right|^{2}} .
\end{aligned}
$$

Upon calculation, we obtain

$$
\begin{aligned}
& A[1]=g_{1}\left(1-\frac{4 h C_{1}}{g_{1} \Gamma}\right), \\
& B[1]=g_{2}\left(1-\frac{4 h C_{2}}{g_{2} \Gamma}\right) .
\end{aligned}
$$


Here

$$
\begin{aligned}
C_{1}= & -e^{2 \vartheta} \varrho^{+} g_{1}\left(\gamma^{*} \beta+\alpha^{*} \delta\right)-e^{-2 \vartheta} \varrho^{-} g_{1}\left(\gamma \beta^{*}+\alpha \delta^{*}\right) \\
& -\varrho^{+}\left[g_{1}\left(|\beta|^{2}+|\alpha|^{2}\right)+g_{2}\left(\beta^{*} \delta-\gamma^{*} \alpha\right)\right] \\
& -\varrho^{-}\left[g_{1}\left(|\gamma|^{2}+|\delta|^{2}\right)+g_{2}\left(\alpha \gamma^{*}-\beta^{*} \delta\right)\right], \\
C_{2}= & -e^{2 \vartheta} \varrho^{+} g_{1}\left(\gamma^{*} \beta+\alpha^{*} \delta\right)-e^{-2 \vartheta} \varrho^{-} g_{2}\left(\gamma \beta^{*}+\alpha \delta^{*}\right) \\
& -\varrho^{+}\left[g_{2}\left(|\gamma|^{2}+|\delta|^{2}\right)+g_{1}\left(\beta \delta^{*}-\gamma \alpha^{*}\right)\right] \\
& -\varrho^{-}\left[g_{2}\left(|\beta|^{2}+|\alpha|^{2}\right)+g_{1}\left(\alpha^{*} \gamma-\beta \delta^{*}\right)\right] \\
\Gamma= & e^{2 \vartheta}\left(\delta \alpha^{*}+\beta \gamma^{*}\right)\left[1+\varrho^{+} \varrho^{+}\left(\left|g_{1}\right|^{2}+\left|g_{2}\right|^{2}\right)\right] \\
& +e^{-2 \vartheta}\left(\delta^{*} \alpha+\beta^{*} \gamma\right)\left[1+\varrho^{-} \varrho^{-}\left(\left|g_{1}\right|^{2}+\left|g_{2}\right|^{2}\right)\right] \\
& +\left(|\alpha|^{2}+|\gamma|^{2}+|\delta|^{2}+|\beta|^{2}\right)\left[1+\varrho^{+} \varrho^{-}\left(\left|g_{1}\right|^{2}+\left|g_{2}\right|^{2}\right)\right] .
\end{aligned}
$$

With parameters $\alpha=\beta=\delta=g_{1}=g_{2}=1, \gamma=0$ and $h=1$, we obtain periodic solutions of equation (3.48-3.49) shown in figure 6 .
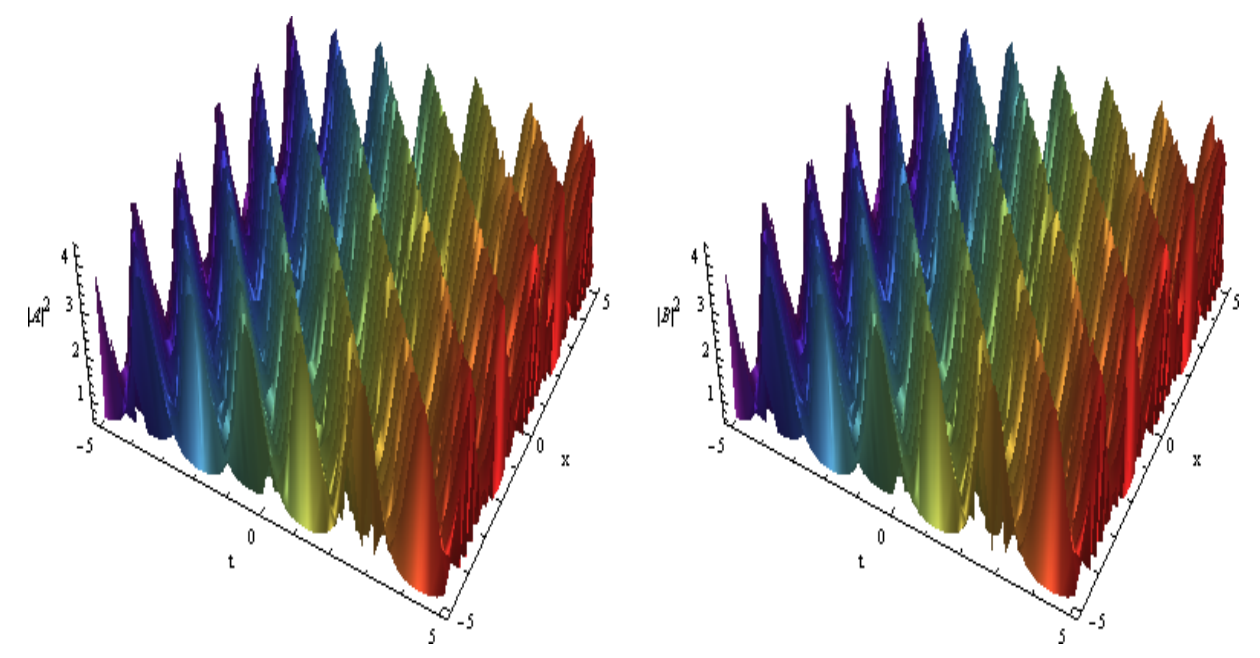

Figure:6 parameters $\alpha=\beta=\delta=g_{1}=g_{2}=1, \gamma=0$ and $h=1$

3.3. Case 2. In this case following generalized DT we get rogue waves solutions of coupled $(\mathrm{cmKdV})$ system. We start with plane wave seed solution

$$
\begin{aligned}
& A=g_{1} e^{i \epsilon_{1}}, \quad \epsilon_{1}=f_{1} x+\left[f_{1}^{3}-6\left(g_{1}^{2}+g_{2}^{2}\right) f_{1}\right] t \\
& B=g_{2} e^{i \epsilon_{2}}, \quad \epsilon_{2}=f_{2} x+\left[f_{2}^{3}-6\left(g_{1}^{2}+g_{2}^{2}\right) f_{2}\right] t
\end{aligned}
$$


We get special solution as after inserting

$$
\Psi(\lambda)=\left(\begin{array}{c}
\alpha e^{i \chi_{1}}+\beta e^{i \chi_{2}} \\
\left(\alpha \delta e^{i \chi_{1}}-\beta \delta e^{i \chi_{2}}\right) e^{i\left(\epsilon_{1}-\epsilon_{2}\right)} \\
\left(\alpha \frac{-g_{1}-g_{2} \delta}{i f_{1}+i \tau_{1}-i \lambda} e^{i \chi_{1}}+\beta \frac{-g_{1}-g_{2} \delta}{i f_{1}+i \tau_{2}-i \lambda} e^{i \chi_{2}}\right) e^{i \epsilon_{1}} \\
\left(\alpha \frac{-g_{2}-g_{1} \delta}{i f_{2}+i \tau_{1}-i \lambda} e^{i \chi_{1}}+\beta \frac{-g_{2}-g_{1} \delta}{i f_{2}+i \tau_{2}-i \lambda} e^{i \chi_{2}}\right) e^{-i \epsilon_{2}}
\end{array}\right)
$$

Where

$$
\begin{aligned}
\chi_{i}= & {\left[\tau_{i} x+\tau_{i}^{3}-3 \lambda \tau_{i}^{2}+\left(3 \lambda^{3}-3 g_{1}^{2}-3 g_{2}^{2}\right) \tau_{i}+3 \lambda^{3}+3\left(g_{1}^{2}+g_{2}^{2}\right) \lambda+3\left(f_{1} g_{1}^{2}-f_{2} g_{2}^{2}\right)\right.} \\
& \left.+3 \delta g_{1} g_{2}\left(f_{1}+f_{2}\right)\right] t
\end{aligned}
$$

$\tau_{i}$ satisfy as

$$
\begin{aligned}
\tau_{i}+\lambda-g_{1} \frac{g_{1}+g_{2} \delta}{f_{1}+\tau_{i}-\lambda}-g_{2} \frac{g_{2}-g_{1} \delta}{-f_{2}+\tau_{i}-\lambda} & =0, \\
\delta\left(f_{1}-f_{2}+\tau_{i}+\lambda\right)-g_{2} \frac{g_{1}+g_{2} \delta}{f_{1}+\tau_{i}-\lambda}+g_{1} \frac{g_{2}+g_{1} \delta}{-f_{2}+\tau_{i}-\lambda} & =0 .
\end{aligned}
$$

With values $g_{1}=1, g_{2}=\frac{1}{2}, f_{1}=\frac{3}{2}, f_{2}=\frac{-3}{2}, \delta=0$ the seed solution in equation (3.53) changed to

$$
A=e^{\frac{3}{8} i(4 x-21 t)}, \quad B=\frac{1}{2} e^{-\frac{3}{8} i(4 x-21 t)}
$$

Now the solution in equation (3.54) takes the form as

$$
\Psi(\lambda)=\left(\begin{array}{c}
e^{-\frac{16}{3} i(4 x-21 t)}\left(\alpha e^{P}+\beta e^{-P}\right) \\
0 \\
e^{\frac{16}{3} i(4 x-21 t)}\left(\alpha \tau_{1} e^{P}+\beta \tau_{2} e^{-P}\right) \\
-\frac{1}{2} e^{\frac{16}{3} i(4 x-21 t)}\left(\alpha \tau_{1} e^{P}+\beta \tau_{2} e^{-P}\right)
\end{array}\right)
$$

Here

$$
\begin{aligned}
\tau_{1} & =\frac{4 i}{3+\sqrt{16 \lambda^{2}-24 \lambda+29}-4 \lambda} \\
\tau_{2} & =\frac{-4 i}{-3+\sqrt{16 \lambda^{2}-24 \lambda+29}+4 \lambda} \\
P & =\frac{1}{4} \sqrt{16 \lambda^{2}-24 \lambda+29} i\left[x+\left(4 \lambda^{2}+3 \lambda-\frac{1}{4}\right) t\right] .
\end{aligned}
$$

Suppose $\lambda_{1}=\frac{3}{4}-\frac{\sqrt{5}}{2} i$ and expanding vector $\Psi\left(\lambda_{1}+\nu^{2}\right)$ in (3.57) with $\nu=0$ we get generalized DT as

$$
\Psi=\Psi^{[0]}+\Psi^{[1]} \nu^{2}+\Psi^{[2]} \nu^{4}+, \ldots \ldots \ldots \ldots \ldots+\Psi^{[N]} \nu^{2 N}+, \ldots \ldots \ldots,
$$

Here $\Psi^{[i]}=\left.\frac{1}{(2 i) !} \frac{\partial^{(2 i)}}{\partial \nu^{(2 i)}}\right|_{\nu \rightarrow 0} \Psi(\nu), \quad(i=0,1,2, \ldots .)$.

By the following limit process

$$
\Psi[1]=\lim _{\nu \rightarrow 0} \frac{\left.B_{\Lambda, \sigma}\right|_{\lambda=\lambda_{1}+\nu^{2 \Psi}}}{\nu^{2}}+\left.B_{\Lambda, \sigma}\right|_{\lambda=\lambda_{1}} \Psi[1]+\frac{\Lambda \Upsilon(\Lambda, \Lambda)^{-1} \Upsilon(\Psi[0], \Lambda)}{\lambda_{1}-\lambda_{1}^{*}}
$$


With $\lambda_{1}=\frac{3}{4}-\frac{\sqrt{5}}{2} i$ and generalized DT inserting these values into (3.27) we obtain vector rogue waves solution of coupled (cmKdV) of first order with $\alpha=1, \beta=0$, as

$$
\begin{aligned}
& A[1]=e^{\frac{3}{8} i(4 x-21 t)}\left(1-\frac{-1440 i t+64}{Q}\right), \\
& B[1]=\frac{1}{2} e^{-\frac{3}{8} i(4 x-21 t)}\left(1-\frac{-1440 i t+64}{Q}\right) .
\end{aligned}
$$

Here

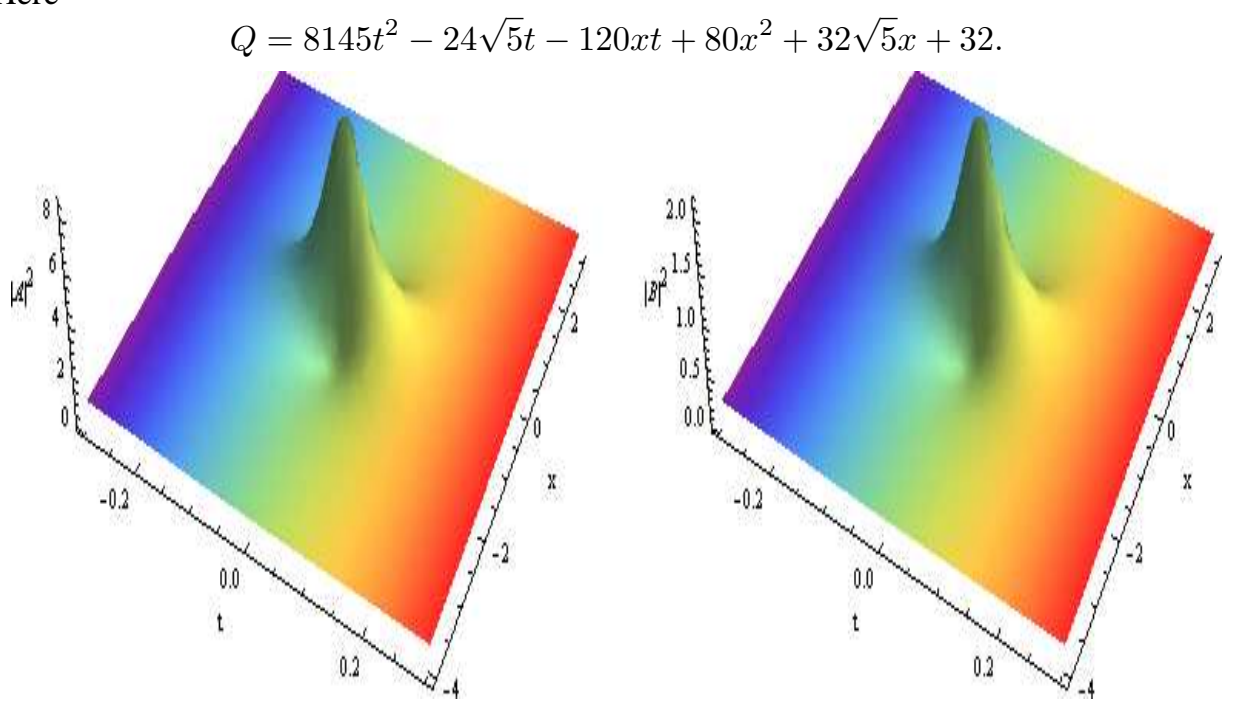

Figure:7 First-order rouge waves having parameters $\alpha=g_{1}=1, g_{2}=\frac{1}{2}, \beta=\delta=$ $0, f_{1}=-f_{2}=\frac{3}{2}$

Any one can be obtain higher-order solitons with similar fashion.

\section{CONCLUDING REMARKS}

In this research work, we have obtained various types of solutions of famous coupled $(\mathrm{cmKdV})$ integrable system. For obtaining solutions, we have used binary DT technique. We have obtained zero seed and non-zero seed solutions. Their classification involved, breather solution and one soliton solution in first case, while in non-zero seed solutions, we obtained bright-bright, w-shaped, bright-dark, periodic and rogue waves solutions. In section 3 , we have obtained only one soliton solutions when $N=1$ and rogue waves having order one. We shall try to obtain higher-order soliton solutions and rogue waves in next publications.

\section{REFERENCES}

[1] B. Alexey, V. S. Matveev, E. Miranda and S. Tabachnikov, Open problems, questions and challenges in finite-dimensional integrable systems, Philosophical Transactions of the Royal Society A: Mathematical, Physical and Engineering Sciences, 376, No. 2131 (2018) 20170430.

[2] M. R. Ali, Solution of KdV and boussinesq using Darboux transformation, Communication in Mathematical Modeling and Applications, 3, No. 3 (2018) 16-27. 
[3] B. Ali, N. M. Yagmurlu, Y. Ucasr and A. Esen, A new perspective for the numerical solutions of the cmKdV equation via modified cubic B-spline differential quadrature method, International Journal of Modern Physics, C 29, No. 06 (2018) 1850043.

[4] S. Bertrand, On integrability aspects of the supersymmetric sine-Gordon equation, Journal of Physics A: Mathematical and Theoretical, 50, No. 16 (2017) 165202

[5] J. Cheng and D. E. Pelinovsky, Rogue periodic waves of the modified KdV equation, Nonlinearity, 31, No. 5 (2018) 1955.

[6] R. I. Ivanov, Two-component integrable systems modelling shallow water waves: the constant vorticity case, Wave Motion, 46, No. 6 (2009) 389-396.

[7] B. A. Kupershmidt, Mathematics of dispersive water waves. Communications in Mathematical Physics, 99, No. 1 (1985) 51-73.

[8] W. Liu, Y. Zhang, and J. He, Dynamics of the smooth positons of the complex modified KdV equation, Waves in Random and Complex Media 28, No. 2 (2018): 203-214.

[9] L.J. Liu, and X. Yu, Solitons and breathers for nonisospectral mKdV equation with Darboux transformation, arXiv preprint arXiv:1710.05108 (2017).

[10] L. Y. Ma, Shou-Feng Shen, and Zuo-Nong Zhu. Soliton solution and gauge equivalence for an integrable nonlocal complex modified Korteweg-de Vries equation, Journal of Mathematical Physics, 58, No. 10 (2017): 103501.

[11] W. X. Ma,Riemann-Hilbert problems and soliton solutions of a multicomponent $m$ KdV system and its reduction, Mathematical Methods in the Applied Sciences, 42, No. 4 (2019) 1099-1113.

[12] W. X. Ma, Y. Zhang and Y. Tang. Symbolic computation of lump solutions to a combined equation involving three types of nonlinear terms, East Asian J Appl Math. 10, No. 4 (2020) 732-745.

[13] W.X. Ma, Binary Darboux transformation for general matrix $m K d V$ equations and reduced counterparts, Chaos, Solitons \& Fractals, 146 (2021) 110824.

[14] W.X. Ma, and S. Batwa, A binary Darboux transformation for multicomponent NLS equations and their reductions, Analysis and Mathematical Physics, 11, No. 2 (2021) 1-12.

[15] Y. Nobuo, and M. Oikawa. A class of exactly solvable nonlinear evolution equations, Progress of Theoretical Physics, 54, No. 5 (1975) 1576-1577.

[16] E.N. Pelinovsky, E. G. Shurgalina, A. V. Sergeeva, T. G. Talipova, G. A. El, and R. H. J. Grimshaw, Twosoliton interaction as an elementary act of soliton turbulence in integrable systems. Physics Letters A, 377, No. 3-4 (2013) 272-275.

[17] H. Riaz, A. Wajahat, and M. ul Hassan, Darboux transformation of a semi-discrete coupled dispersionless integrable system, Communications in Nonlinear Science and Numerical Simulation, 48 (2017) 387-397.

[18] C. Rogers and P. A. Clarkson, Ermakov-Painlev é II reduction in cold plasma physics. Application of a Bäcklund transformation, Journal of nonlinear mathematical physics, 25, No. 2 (2018) 247-261.

[19] N. Sasa, and J. Satsuma, New-type of soliton solutions for a higher-order nonlinear Schrödinger equation. Journal of the Physical Society of Japan, 60, No. 2 (1991) 409-417.

[20] A. V. Slunyaev and E. N. Pelinovsky, Role of multiple soliton interactions in the generation of rogue waves: the modified Korteweg-de Vries framework, Physical review letters, 117, No. 21 (2016) 214501.

[21] P. Suret, A. Picozzi and S. Randoux, Wave turbulence in integrable systems: nonlinear propagation of incoherent optical waves in single-mode fibers, Optics express 19, No. 18 (2011): 17852-17863.

[22] F. S. Tian, Initial-boundary value problems of the coupled modified Korteweg-de Vries equation on the halfline via the Fokas method, Journal of Physics A: Mathematical and Theoretical, 50, No. 39 (2017) 395204.

[23] A. M. Tsvelik, Incommensurate phases of quantum one-dimensional magnetics, Physical Review B, 42, No. 1 (1990) 779

[24] D. S. Wang, D.J. Zhang and J. Yang, Integrable properties of the general coupled nonlinear Schrödinger equations, Journal of Mathematical Physics, 51, No. 2 (2010): 023510.

[25] P. Walczak, S. Randoux, and P. Suret, Optical rogue waves in integrable turbulence, Physical review letters, 114, No. 14 (2015) 143903.

[26] X.Y. Wen and X. Yong, Higher-order rogue wave and rational soliton solutions of discrete complex $m$ KdV equations, East Asian J. Appl. Math. 8 (2018) 100.

[27] T. Xu, L. Hengji, H. Zhang, M. Li, and S. Lan, Darboux transformation and analytic solutions of the discrete PT-symmetric nonlocal nonlinear Schrödinger equation, Applied Mathematics Letters, 63 (2017) 88-94. 
[28] J. L. Yan, and L. H. Zheng, Conservative finite volume element schemes for the complex modified Kortewegde Vries equation, International Journal of Applied Mathematics and Computer Science, 27, No. 3 (2017) 515-525.

[29] J. Y. Yang, W. X. Ma, and C. M. Khalique, Determining lump solutions for a combined soliton equation in $(2+1)$-dimensions, The European Physical Journal Plus, 135, No. 6 (2020) 1-13.

[30] Q. H. Zhang and F. Chen. Dark and antidark solitons for the defocusing coupled Sasa-Satsuma system by the Darboux transformation, Applied Mathematics Letters, 88 (2019) 237-242.

[31] H. Q. Zhang, Y. Wang, and Wen-Xiu Ma. Binary Darboux transformation for the coupled Sasa-Satsuma equations, Chaos: An Interdisciplinary Journal of Nonlinear Science, 27, No. 7 (2017) 073102. 